\title{
Perceptual selection based on alphanumeric class: Evidence from partial reports
}

\author{
JOHN DUNCAN \\ MRC Applied Psychology Unit, Cambridge, England
}

\begin{abstract}
In two experiments, subjects were shown brief displays of mixed digits and letters. In partial report, the task was to report as many digits as possible while ignoring letters, or vice versa. In whole report, both digits and letters were to be reported. There were two main findings. The probability of reporting digits (letters) was greater when letters (digits) could be ignored (partial report) than when they could not (whole report). When letters (digits) could be ignored, the probability of reporting a given digit (letter) increased as the number of digits (letters) in the display decreased. Both findings held even at exposure durations so brief that only two or three characters were ever reported, minimizing the chance of output interference. While the tendency was for hit rates and false alarm rates to covary, the major findings held even in the ROC curves of individual subjects, and so were not due to simple criterion shifts. The results suggest that digits and letters can be separated "preattentively," that is, prior to a limited-capacity system (LCS) in visual perception. Thus, in partial report, the main competition for LCS comes from characters in the target class, nontargets being rejected at a prior level.
\end{abstract}

\section{Theories of Visual Attention}

Suppose a group of stimuli (e.g., a row of letters) is briefly shown in a tachistoscope. At a suitable exposure duration, the observer may be able to report only one or two of the stimuli, yet may choose reliably which one or two (e.g., the leftmost) these are to be (Allport, 1971; Sperling, 1967). Phenomenally, the observer "pays attention" to some stimuli and not to others. Thus, theories of "attention" in visual perception concern a system severely limited in its capacity to deal with several stimuli at once, yet capable of direction to one chosen stimulus or another. Here it will be termed the "limitedcapacity system"' (LCS).

Theories of attention typically assume that the access of chosen stimuli to LCS follows a preliminary, "preattentive" stage of perceptual analysis, acting in parallel across simultaneous stimuli. The nature of preattentive analysis is a matter of dispute. Some theories suppose it to concern only simple stimulus characteristics such as location or color, leaving the detailed analysis of form to take place in LCS itself (Broadbent, 1971). Others envision a much more complete preattentive analysis, concerning even details of form and aspects of meaning (Allport, 1977; Posner, 1978). These two positions are extremes: other attempts to delimit the nature of preattentive processing have also been made (e.g., Treisman \& Gelade, 1980).

Recently, I reported a set of experiments in support of the view that even form and meaning can

The author's mailing address is: MRC Applied Psychology Unit, 15 Chaucer Road, Cambridge CB2 2EF, England. be analyzed preattentively (Duncan, 1980). Subjects searched displays of letter nontargets for digit targets. Each display had four characters, arranged in the form of a plus sign centered on fixation. Characters at 12 and 6 o'clock made up the "vertical limb" of the plus, while those at 9 and 3 o'clock made up the "horizontal limb." Digit targets occurred independently and with a probability of onethird in each limb, so that in a single display there could be targets in either, neither, or both limbs. Separate keys were to be pressed for targets detected in the two limbs, so that on a single trial the subject might press either, neither, or both keys. Exposures were brief, and accuracy was the dependent measure.

A critical factor concerned the way trials were classified in scoring the data. Consider the process of scoring accuracy on the vertical limb, A. Various types of trial could be distinguished according to simultaneous events on the horizontal limb, B. In total there were four types of trial-those with, on B, a hit (target presented; detection response made), false alarm (no target presented; detection response made), miss (target presented; no detection response made), or correct rejection (no target presented; no detection response made)-although, in fact, there were sufficient data to consider only two types, those with $B$ hits and B correct rejections. It emerged that performance on $A$ was much better when B had a correct rejection than when $B$ had a hit. Indeed, when $B$ had a correct rejection, performance on A was almost comparable to performance in a control condition in which only $A$ was displayed.

Exactly the same pattern emerged when the analy- 
sis was reversed: Performance on B was much better when $\mathbf{A}$ had a correct rejection than when $\mathbf{A}$ had a hit. A similar pattern of results has also been seen in a variety of auditory tasks (Moray, Fitter, Ostry, Favreau, \& Nagy, 1976; Ostry, Moray, \& Marks, 1976; Sorkin, Pohlmann, \& Gilliom, 1973).

The theory developed to account for these results is illustrated in Figure 1. Four alphanumeric characters, A5TX, are assumed to be present in the visual field. (No attempt has been made to represent their spatial arrangement.) Two levels of the perceptual system are distinguished. The first (preattentive) level operates in parallel across simultaneous stimuli. Here each stimulus is fully identified, in form, color, size, position, and so on, and already much well-learned information (e.g., status as letter or digit) is derived from memory. However, no information can yet serve as the basis for report. Phenomenally, nothing has yet reached awareness. To be reported, a stimulus representation formed at the first level must pass through LCS to the second level. Phenomenally, attention must be directed to the stimulus.

LCS cannot deal effectively with more than one stimulus at a time. A selection schedule determines which stimuli LCS is to receive. Potentially, any information derived at the first level might serve as the basis for selection. Thus, the subject might wish to report stimuli in a given position, or stimuli of a given color, and so on. In the task described above, however, we should assume that selection is based on alphanumeric class: Digit targets pass into LCS, while letter nontargets are withheld (cf. Figure 1).

The account of the data is as follows. A hit on limb B suggests that a target from B occupies LCS.

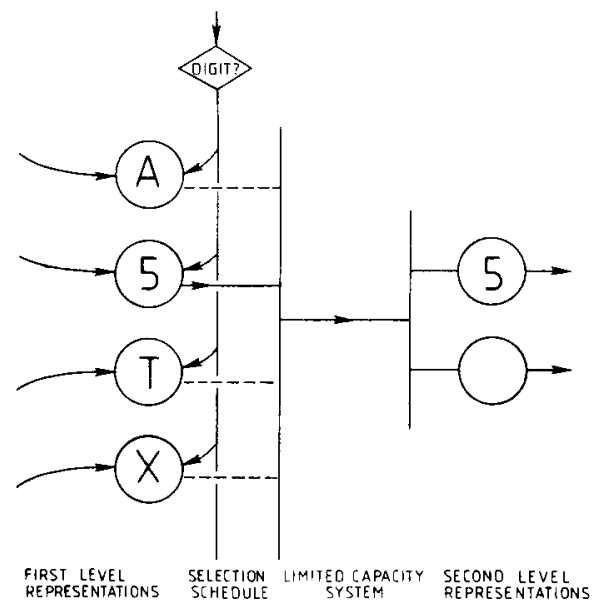

Figure 1. Two-level perceptual representation of four alphanumeric characters present in the visual field. Although only shape information is shown, the representation of each stimulus would include also information concerning position, color, size, and so on.
It follows that targets in limb A will be hard to detect, since LCS cannot without loss pass two stimuli at once to the second level. Performance on A will be poor. On the other hand, a correct rejection on B suggests that no stimulus from B occupies LCS. It follows that performance on $A$ will be good.

The crucial point is that access to LCS can apparently be determined by a character's status as digit or letter. The similar results of Ostry et al. (1976), obtained in an auditory task in which targets were animal names and nontargets were other words, suggest that even the meaning of a word can determine LCS access. It is for this reason that the theory proposes a full preattentive analysis of form and meaning. LCS is seen as a system which outputs the completed results of perceptual analysis to a further level necessary for report and, phenomenally, awareness. This may be termed the final transfer theory.

\section{Partial Report}

The proposal of concern is that access to LCS can be determined by alphanumeric class. One relevant line of evidence comes from work with the partial report task of Sperling (1960).

In a typical experiment, displays might each contain three digits and three letters in random order. In the partial report condition, the subject must report as many digits as possible while ignoring letters, or as many letters as possible while ignoring digits. In the whole report condition, both digits and letters are to be reported.

Hit rate for digits may be taken as the probability, given that a digit is present in the display, that it will be reported-and similarly for letters. The early results of Sperling (1960) and von Wright (1970) suggested equal hit rates in partial report and whole report, at least for most subjects. Thus, the probability of reporting digits (letters) was no greater when letters (digits) could be ignored than when they could not. Later work suggests that hit rates are a little higher in partial report than in whole report (Dick, 1969, 1970, 1971), especially if the target class (digits or letters) in partial report is specified well in advance of the stimulus presentation (Merikle, 1980; von Wright, 1972). However, the difference (termed partial report superiority) is small, and certainly not comparable to the large effect seen when targets in partial report are defined by position, for example, reporting letters from the top one of two rows of three, or color, for example, reporting red letters from a mixed array of three red and three black (Sperling, 1960; von Wright, 1968, 1970).

Predictions from the final transfer theory are influenced by two separate factors. First is the number of stimuli competing for LCS, three in partial report but six in whole report. Considering this 
factor alone, one might expect a large partial report superiority even when targets are defined by alphanumeric class, comparable to that seen when they are defined by position or color. The clarity of this prediction is obscured, however, by uncertainty over the influence of the second factor, the selection schedule in force for each task.

Performance in partial report always depends on the particular stimulus property used to distinguish targets from nontargets. For example, selection based on position can be better than selection based on color, and so on (von Wright, 1968, 1972). Similarly, the efficiency of selection based on a particular dimension (e.g., color) depends on the discriminability between targets and nontargets along that dimension (von Wright, 1970). This is as we should expect. At the first level, different stimulus properties (position, color, alphanumeric class) will be derived with different speed and accuracy, and will be differentially effective in guiding LCS access (Duncan, 1980, 1981). Performance in general will be a joint function of the number of stimuli competing for LCS, and the particular selection schedule in force.

The selection schedule used in whole report is unknown: quite possibly it varies. Often subjects may spontaneously adopt a strategy based on position, reading stimuli from the display in a fixed spatial order. But, in any case, selection schedules will usually be different in partial report and whole report. As the possible influence of this difference in selection schedules cannot be assessed, neither can absolute differences in performance be predicted. Previous data suggest that, when targets in partial report are defined by alphanumeric class, a slight advantage over whole report is the rule.

\section{Aims of the Present Research}

The present research was designed to extend previous partial report work in four ways:

(1) An important prediction of the final transfer theory concerns the size of partial report superiority as a function of the number of targets in the display.

Let a total display of $n$ characters consist of $d$ digits and $n-d$ letters. In partial report, if only digits are to be reported, hit rate should increase as $d$, and hence the number of characters competing for LCS, decreases. In whole report, on the other hand, hit rate should be independent of $d$, since digits and letters compete for LCS equally.

In the present Experiment 1, displays consisted of either one digit and five letters or three digits and three letters. Digits were the targets in partial report. Predictions were that: (a) partial reports should be better with one-digit displays than with three-digit displays; (b) whole reports should be relatively independent of display type; and (c) hence, partial report superiority should be greater with one-digit displays.

Such findings would be quite analogous to the results of Duncan (1980). They would show that, in partial report, the probability of reporting a given digit is greater when this digit shares the display with five letters than when it shares the display with two other digits and three letters. Again this would suggest preferential access of digit targets to LCS.

(2) An omission in previous partial report work concerns false alarms, that is, cases in which a character not present in the display is falsely reported. Data have typically been presented as the product of hit rate and display size, an estimate of the "total number of characters available." False alarms have been ignored (Dick, 1969, 1970, 1971; Merikle, 1980; Sperling, 1960; von Wright, 1970, 1972).

False alarms may be of interest in their own right. Furthermore, it is accepted that varying hit rates cannot be clearly interpreted without knowledge of false alarm rates. The present work considers both hit rates and false alarm rates.

(3) Most previous partial report work has been motivated by an interest in iconic memory. Accordingly, backward masks have not been used to control the time for which stimulus information is usefully available, and the number of characters reported per trial has been correspondingly large, up to four or five.

For present purposes, a disadvantage of this procedure concerns the possibility of output interference. It is sometimes suggested (e.g. Dick, 1971) that the advantage of partial report over whole report may be due to output processes. Since, in whole report, there is a greater total number of characters to be stored and reported, there may be a greater chance that any one will be lost while others are output.

Presumably, the importance of output interference may be minimized by reducing exposure duration to the point at which only two or three characters are ever reported. Under these circumstances, it seems likely that performance will be limited entirely by processes taking place at the time of stimulus input. In the present work, performance was examined at a range of exposure durations, both with and without backward masks.

(4) A final point concerns the amount of perceptual analysis needed to distinguish a set of digits from a set of letters. In some cases, knowledge of only a few key stimulus features might allow a fairly successful separation.

An extreme case concerns an earlier partial report experiment of mine (Duncan, Note 1) in which digits were drawn from the set 23456789 and letters from the set CEJLNPUX. In these sets, only one digit (4) has a complete or almost complete ver- 
tical line, as contrasted with all but two letters (C, $X)$. Analysis solely for the presence of a vertical line could separate the two sets quite successfully. ${ }^{1}$ (Other workers have used similar sets in related experiments. For example, Taylor (1978) used the sets 23456789 and CDFHNRTX, while Schneider and Shiffrin (1977) used 123456789 and CDFGHJKLM.)

In the present work, three character sets were used. In Set 1 were the digits 134568 and the letters CJPQXY. These two groups were chosen to be matched, as carefully as possible, on the following characteristics: presence of curves, presence of closures, presence of complete vertical lines, presence of complete horizontal lines, vertical symmetry, horizontal symmetry, "left-facing" appearance, "right-facing" appearance, mean total length of character contour. Thus, in set 1 , every attempt was made to ensure that no single component or feature of the characters could reliably separate the digits from the letters. Letters were also chosen so that, as far as possible, none was highly confusable with any particular digit.

In set 2 were the digits 235678 and the letters EJLNPU. These were a subset of the characters used before (Duncan, Note 1), chosen to have a complete or almost complete vertical line in every letter but in no digit. Large differences from performance with set 1 might indicate separation of digits and letters based on vertical lines alone.

In set 3 were the digits 245678 and the letters ABGSTZ. For each digit there was at least one highly confusable letter (Poulton, 1968). Confusable pairs were 2-Z, 4-A, 5-S, 6-G, 7-T, 8-B. Pilot work had suggested that confusability could influence partial reports.

\section{Conceptualization of the Discrimination Process}

The conceptualization. The aim of this section is to present a conceptualization of the discrimination process in partial report and whole report which leads to useful performance scores. In particular, we need scores that allow direct comparisons between performance with displays containing one digit and five letters and performance with displays containing three digits and three letters. Two details of experimental procedure are important. Subjects never knew how many digits or letters a particular display would contain, and were free to report as many as they chose. In making their report, they gave only character identities, not positions.

Again, let a total display of $\mathbf{n}$ characters contain $\mathrm{d}$ digits and $\mathrm{n}-\mathrm{d}$ letters. The goal is a performance measure which, for the ideal observer, would be independent of $\mathrm{d}$; allowing deviations from independence to be ascribed to the processing constraints of the psychological subject.

An unsuitable measure is the probability, given that a response is made, that it will be correct. If the entire character set used in the experiment contains $\mathrm{D}$ digits and $\mathrm{L}$ letters, then the probability correct for a character guessed at random is $d / D$ for a digit and $(n-d) / L$ for a letter. For this measure, scores of the ideal observer could not be independent of $d$.

The problem is avoided by basing scores on a different probability: the probability, given that a character is or is not present in the display, that it will appear in the report. It is useful to consider first a simplified task. On each trial, the subject is shown a display of $\mathrm{n}$ characters, and must simply decide whether or not a particular character $\alpha$ is present. We may assume that, from each display, the ideal observer derives a particular value of evidence in favor of $\alpha$, and reports $\alpha$ 's presence whenever this value exceeds a criterion. Natural measures of performance are hit rate, or the probability, given that $\alpha$ is present in the display, that it will be reported as present, and false alarm rate, or the probability, given that $\alpha$ is absent from the display, that it will be reported as present. Providing the average confusability between $\alpha$ and digits equals the average confusability between $\alpha$ and letters, performance will be independent of $d$. The probability that evidence in favor of $\alpha$ exceeds criterion, when $\alpha$ is or is not present in the display, will not depend on whether other displayed characters are digits or letters. (Note again that the observer does not know how many digits or letters will be present, so that identification of one character gives no information about others. Relaxation of the assumption concerning equal confusability with digits and letters will be considered later in the paper.)

Generalization to the tasks of the present research is achieved as follows. It is assumed that any character from the set used in the experiment is reported when evidence in its favor exceeds criterion. The probability of this, when the character is or is not present in the display, again should not depend on whether other displayed characters are digits or letters. Thus, for the ideal observer, measures of mean hit rate-or the mean probability, given that a character is present in the display, that it will appear in the report-and mean false alarm rate-or the mean probability, given that a character is absent from the display, that it will appear in the reportshould be independent of $d$. Details of the derivation of these scores will be presented after a full description of the experimental method.

Predictions. The above conceptualization allows predictions from the final transfer theory to be derived with increased clarity.

Consider the process of deciding whether or not a particular character $\alpha$ is present in each display. At the first level, that is, prior to LCS, we assume a continuous scale of evidence in favor of $\alpha$, with the evidence for $\alpha$ on a particular trial represented 
by a point somewhere along this scale. Thus, two distributions may be drawn on the scale, one for trials with $\alpha$ actually present in the display and one for trials with $\alpha$ actually absent.

[In fact, it may be useful to think that, on each trial, each character in the display gives a separate value of evidence in favor of $\alpha$. Each trial will then have a maximum value of evidence for $\alpha$, that is, the value of that one character whose evidence is strongest. The above distributions could then be taken to be distributions of this maximum (cf. Shaw, 1982). In any case, we must assume that evidence in favor of $\alpha$ at the first level is in the form of evidence that a particular displayed character is $\alpha$, so that, if desired, this particular character can be passed into LCS.]

The ideal strategy would be to report $\alpha$ as present whenever, at the first level, the evidence in favor of $\alpha$ exceeds some criterion. However, on some proportion p of trials, this will not be possible. Although the evidence in favor of $\alpha$ exceeds the criterionthat is, some character in the display is confidently identified as $\alpha$-it will not be possible to pass this character to the second level, since access to LCS cannot be obtained. As before, the value of $p$ will be a joint function of the number of characters competing for LCS and the selection schedule in force.

Predictions concerning hits follow as before. Providing the criterion remains constant, the probability of reporting $\alpha$, given that it is present in the display, will decrease as competition for LCS (and hence p) increases. Thus, hit rate will be higher in partial report than in whole report, and, in partial report, will be higher for displays with one digit than for displays with three digits. Interestingly, the same predictions hold for false alarms, since competition for LCS will also cause loss of trials on which a was confidently but incorrectly identified at the first level. Providing the criterion remains constant, the probability of reporting $\alpha$, given that it is not present in the display, will also decrease as competition for LCS increases. Hit and false alarm rates should covary.

A caution concerns the possibility that criterion might not be constant. In this case, the pattern of results might be disturbed. Nevertheless, the above predictions are those following most straightforwardly from the final transfer theory.

\section{EXPERIMENT 1}

\section{Method}

\section{Subjects}

Twenty-four subjects, aged between 18 and 41 , were recruited from the panel of the Applied Psychology Unit. Sixteen were female.

\section{Displays}

Each stimulus display was made up of six characters, evenly spaced around the perimeter of an imaginary circle centered on fixation, with the first character at 12 o'clock. At the viewing distance of approximately $45 \mathrm{~cm}$, each character subtended a visual angle of $3 / 5 \mathrm{deg}$ vertically $\times 1 / 3 \mathrm{deg}$ horizontally, and was centered 1-1/5 deg from fixation. Each array had either one digit and five letters or three digits and three letters, drawn without replacement from the appropriate set.

Masking displays were of similar dimensions, with a superimposed 0 and + , equal in size to other stimulus characters, in each character position.

Displays were presented on a cathode ray tube (P31 phosphor) under control of a Cambridge Electronic Design computer system. Each character was created within a matrix of 13 (vertical) $\times 9$ (horizontal) dots. A central fixation point was present throughout.

\section{Character Sets}

The experiment involved three different character sets, as previously described. Thus, set 1 contained the characters 134568 / CJPQXY, set 2 contained the characters 235678/EJLNPU, and set 3 contained the characters 245678/ABGSTZ. The subjects were divided at random into three equal groups, each serving with a single character set.

\section{Procedure}

The experiment was run on-line in a semi-darkened room. Each trial was initiated by the subject, who, when adequately fixated, pressed a key to give an immediate flash of the stimulus display. (Maximum onset time for a display of six characters was approximately $2 \mathrm{msec}$.) There were two tasks, partial report and whole report. In partial report, the subject reported as many digits as possible while ignoring letters. In whole report, as many as possible of both digits and letters were to be given. Reports were called out immediately and in any order to the experimenter, who sat behind the subject and wrote reports down. Only character identities were given, not positions. Intertrial intervals were variable, since the subject was free to proceed with the next trial as soon as the report was complete.

Four exposure conditions were used. Displays lasted for 60 , 90 , or $120 \mathrm{msec}$, followed at once by the mask for $500 \mathrm{msec}$; or displays lasted for $120 \mathrm{msec}$ with no mask.

\section{Design}

All variables except for character set were manipulated within subjects. Each subject served in eight blocked experimental conditions, 2 (partial report vs. whole report) $\times 4$ (exposure conditions). In each of two hourly sessions, on different days, the subject performed one block of trials per condition. In the first session, each block had 40 trials, in the second, 76. The only data analyzed were those from the last 72 trials of each secondsession block. The remaining variable, number of digits per display, varied randomly within blocks.

In each session, the eight blocks were grouped by task, so that either the four blocks of partial report preceded the four of whole report, or the reverse. Within each set of four blocks, the order of exposure conditions was the same. Orders of tasks and of exposure conditions were counterbalanced within each group of subjects but, for any one subject, were the same in each session.

For each trial, a new stimulus array was randomly created, except that over the last 72 trials of each second-session block there were the following constraints. There were 36 trials each with one-digit displays and with three-digit displays, in random order. For the 36 trials with one-digit displays, each of the six alternative digits appeared once in each of the six alternative positions. For the 36 trials with three-digit displays, no constraints governed the choice of which digits would be shown or which positions they would occupy.

\section{Instructions}

It was repeatedly emphasized that trials should not be initiated without careful fixation. The subject was to report only charac- 
ters of which he or she was "fairly certain," but otherwise could report any number of characters. It was emphasized that a response of "nothing" was always acceptable if nothing had been seen with sufficient certainty. This was especially emphasized for partial report, since it was explained that the number of digits actually present was varying (though not that it was always either one or three), with often only one digit which "might well sometimes be missed." For whole report, it was explained that digits and letters were equally important.

Reports of characters outside the subject's allotted set were not allowed. When they occurred, the subject was referred to a list of the possible characters, always available on the table nearby.

\section{Scoring}

For each subject, there were 36 scored trials in each of the cells defined by 2 tasks $\times 4$ exposure conditions $\times 2$ display types. Separate scores were obtained for digits in each cell, and for letters in each whole-report cell.

Mean hit rate was defined as the probability, given that any character was present in the display, that it would appear in the report. (For the ideal observer, this would correspond to the mean probability, given that a character was present, that evidence in its favor would exceed criterion.) This could straightforwardly have been obtained by dividing the total number of characters correctly reported by the total number presented. The procedure actually used, however, was a slight modification designed to correct for strong serial position effects (i.e., preferences for reporting characters from particular display positions). For each serial position, a separate measure of hit rate was obtained as above, and the mean was then taken across positions.

Mean false alarm rate was defined as the probability, given that any character was absent from the display, that it would appear in the report. (For the ideal observer, this would correspond to the mean probability, given that a character was absent, that evidence in its favor would exceed criterion.) This score was obtained by dividing the mean number of false alarms per trial by the number of characters which could have been falsely reported, that is, the number absent from the display (five and one for digits and letters, respectively, in one-digit displays; three and three, respectively, for three-digit displays).

\section{Results}

Figure 2 shows mean hit rates. There is a separate panel for each type of display (one-digit vs. three- digit), for each of the three character sets. Each panel shows data for three different types of report (digits in partial report, digits in whole report, letters in whole report) as a function of exposure conditions. Corresponding false alarm rates are shown in Table 1.

As results were a little different for cases with and without a backward mask, the two will be considered separately.

\section{Unmasked Case}

Digits in partial report and whole report. The "no-mask" hit data in Figure 2 show that results conformed to predictions. Partial reports were superior to whole reports. In partial report, hit rate was higher for one-digit displays than for threedigit displays, whereas, in whole report, there was no such effect. Thus, partial report superiority was also greater for one-digit displays than for threedigit displays.

The data were examined by analysis of variance (ANOVA), with character set as a between-subjects factor and with task (partial report vs. whole report) and display type (one-digit vs. three-digit) as withinsubjects factors. Character set was significant as a main effect $[F(2,21)=7.0, p<.005]$ but showed no significant interactions. In confirmation of predictions, there were significant effects of task $[F(1,21)=110.6, p<.001]$, display type $[F(1,21)=$ $37.0, \mathrm{p}<.001]$, and task $\times$ display type $[\mathrm{F}(1,21)$ $=21.0, \mathrm{p}<.001]$.

Corresponding "no-mask" false alarm data (Table 1) show that here the predicted results were not obtained. Except for character set 3, false alarm rates were not higher in partial report than in whole report; nor, in partial report, were they higher for one-digit displays than for three-digit displays. The data were examined by ANOVA, as before. There

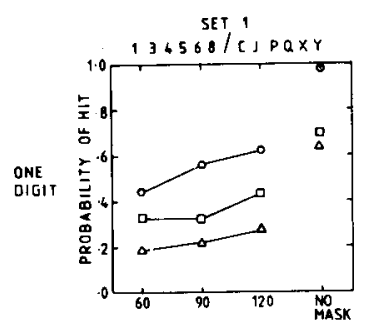

EXPOSURE DURATION (MSEC)

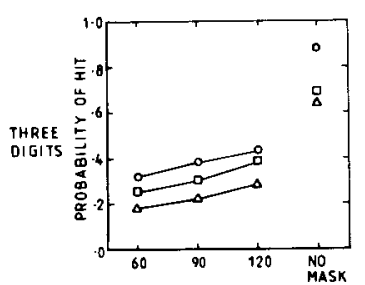

EXPOSURE OURATIONIMSECI

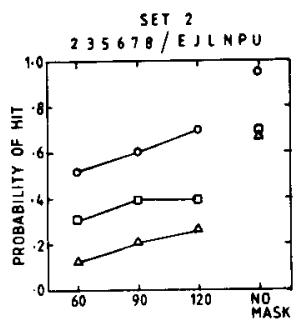

EXPOSURE DURATION (MSEC)

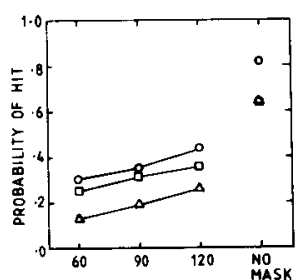

EXPOSURE DURATION (MSEC)

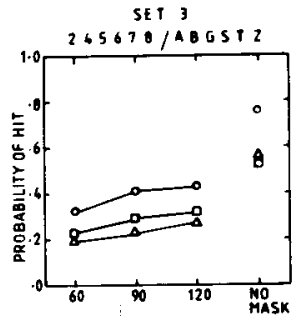

EXPOSURE DURATION (MSEC)

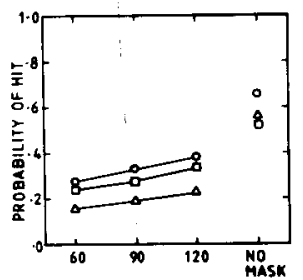

EXPOSURE OURATION IMSECI

Figure 2. Experiment 1. Hit rates in each condition. $O_{-}$, digits in partial report. $\square-$, digits in whole report. $\Delta$-, letters in whole report. 
Table 1

Experiment 1. False Alarm Rates

\begin{tabular}{|c|c|c|c|c|c|c|c|c|}
\hline & \multicolumn{8}{|c|}{ Exposure Conditions } \\
\hline & \multicolumn{4}{|c|}{ One-Digjt Displays } & \multicolumn{4}{|c|}{ Three-Digit Displays } \\
\hline & 60 & 90 & 120 & NM & 60 & 90 & 120 & NM \\
\hline & \multicolumn{8}{|c|}{ Set 1} \\
\hline Partial Report/Digits & .0049 & .0077 & .0084 & .0035 & .0035 & .0023 & .0046 & .0035 \\
\hline Whole Report/Digits & .0028 & .0028 & .0035 & .0049 & .0012 & .0000 & .0023 & .0093 \\
\hline \multirow[t]{2}{*}{ Whole Report/Letters } & .0035 & .0035 & .0035 & .0139 & .0012 & .0035 & .0035 & .0151 \\
\hline & \multicolumn{8}{|c|}{ Set 2} \\
\hline Partial Report/Digits & .0174 & .0229 & .0125 & .0028 & .0162 & .0047 & .0070 & .0035 \\
\hline Whole Report/Digits & .0049 & 0042 & .0042 & .0035 & .0047 & .0012 & .0023 & .0081 \\
\hline \multirow[t]{2}{*}{ Whole Report/Letters } & .0035 & .0000 & .0000 & .0174 & .0000 & .0035 & .0023 & .0197 \\
\hline & \multicolumn{8}{|c|}{ Set 3} \\
\hline Partial Report/Digits & .0208 & .0146 & .0285 & .0292 & .0127 & .0070 & .0081 & .0209 \\
\hline Whole Report/Digits & .0084 & .0090 & .0090 & .0153 & .0058 & .0058 & .0116 & .0104 \\
\hline Whole Report/Letters & .0035 & .0000 & .0104 & .0278 & .0046 & .0070 & .0139 & .0174 \\
\hline
\end{tabular}

Note $-N M=$ no mask.

was a significant effect of character set $[F(2,21)=$ $10.7, \mathrm{p}<.001$ ], reflecting much higher false alarm rates overall for set 3 than for set 1 or set 2. Two significant interactions, character set $\times$ task $[F(2,21)$ $=7.5, \mathrm{p}<.005]$ and character set $\times$ display type $[F(2,21)=4.5, p<.05]$, showed that only for set 3 were false alarm rates higher in partial report than in whole report and higher for one-digit displays than for three-digit displays. A last significant interaction was task $\times$ display type $[\mathrm{F}(1,21)=7.1$, $\mathrm{p}<.05]$. For character sets 1 and 2 , the tendency for false alarm rate to be higher in whole report than in partial report was a little stronger for threedigit displays than for one-digit displays. For character set 3, the tendency for false alarm rate to be lower in whole report than in partial report was a little stronger for one-digit displays than for threedigit displays.

Digits and letters in whole report. The other comparison of interest is between digits and letters in whole report. The "no-mask" data in Figure 2 and Table 1 suggest that, while hit rates for the two were similar, false alarm rates were higher for letters than for digits. Separate ANOVAs for hits and false alarms had character set as a between-subjects factor and character type (digits vs. letters) and display type (one-digit vs. three-digit) as within-subjects factors. The only significant effect was that of character type in the false alarm analysis $[F(1,21)=5.0$, $\mathrm{p}<.05]$.

\section{Masked Case}

Digits in partial report and whole report. The hit data for masked cases in Figure 2 again show a pattern of results approximately as predicted. Partial reports were superior to whole reports. In partial report, hit rate was higher for one-digit dis- plays than for three-digit displays. This time there was a hint of a similar effect even in whole report, but, as the partial report effect was much the stronger, it was again true that partial report superiority was greater for one-digit displays than for three-digit displays.

The ANOVA was similar to that used before, with the addition of exposure duration as a withinsubjects factor. As before, the basic predictions were confirmed by significant effects of task $[F(1,21)=$ $67.0, p<.001]$, display type $[F(1,21)=68.3, p<.001]$, and task $\times$ display type $[F(1,21)=91.8, p<.001]$. This time, however, these basic effects were modified by two further interactions, character set $x$ display type $[F(2,21)=10.0, p<.001]$ and character set $x$ task $\times$ display type $[F(2,21)=8.0, p<.005]$. Especially in partial report, the effect of display type was reduced for character set 3 . Exposure duration was significant as a main effect $[\mathrm{F}(2,42)=86.6, \mathrm{p}<.001]$, but showed no significant interactions.

This time, predictions were also confirmed in the false alarm data. False alarm rates were higher in partial report than in whole report. In partial report, false alarm rates were higher for one-digit displays than for three-digit displays. Again, however, there was a suggestion of a similar, though weaker, effect of display type even in whole report.

The data were examined by ANOVA, as before. There were significant effects of task $[F(1,21)=$ $13.5, \mathrm{p}<.005]$, display type $[\mathrm{F}(1,21)=10.4, \mathrm{p}<$ $.005]$, and task $\times$ display type $[F(1,21)=4.6, p<$ .051 .

Digits and letters in whole report. The data for masked cases in Figure 2 show that, in whole report, hit rates were higher for digits than for letters. This was confirmed by an ANOVA, similar to those described before, which showed a significant effect 
of character type $[F(1,21)=46.5, p<.001]$. Also significant were display type $[F(1,21)=16.7, p<$ $.001]$, reflecting generally higher hit rates for onedigit displays than for three-digit displays, and character set $\times$ character type $\times$ display type $[F(2,21)$ $=5.5, \mathrm{p}<.05]$, reflecting the fact that, for character sets 1 and 2, the effect of display type was carried only by the digits, while for character set 3 , it was carried only by the letters. The last significant effect was exposure duration $[F(2,42)=64.4$, $\mathrm{p}<.001]$

In a similar ANOVA for false alarms, there were no significant effects, reflecting the lack of obvious pattern in the corresponding data of Table 1.

\section{Discussion}

\section{Partial Report Superiority}

Major predictions concerning hits were confirmed with all character sets, and under all exposure conditions. Partial reports were superior to whole reports. In partial report, hit rate was higher for onedigit displays than for three-digit displays. An unexpected result, to be discussed later, was a tendency, even in whole report, for a similar effect of display type. The partial report effect, however, was much the stronger, producing the expected interaction between task and display type.

These results lend support to the view that a character's access to LCS can be determined by alphanumeric class. The probability of reporting digits is greater when letters can be ignored (partial report) than when they cannot (whole report). If letters can be ignored, the probability of reporting a particular digit present in the display is greater when this digit shares the display with five letters than when it shares the display with two other digits and three letters.

Predictions concerning false alarms were confirmed except for character sets 1 and 2 with unmasked presentations. Occasional deviations from the predicted pattern could well be explained by small variations in criterion, as previously discussed.

There is, however, a major difficulty with the argument as it stands. Covarying hit and false alarm rates, while predicted by the final transfer theory, also invite an explanation entirely in terms of criterion shifts. A lower criterion in partial report than in whole report, especially for one-digit displays, could have been the major factor responsible for differences in performance. In accounting for these results, it could be unnecessary to invoke any factor of differential competition for LCS.

The standard solution to this problem is to vary criteria experimentally, producing for each condition an entire receiver operating characteristic $(R O C)$ or plot of hit rate against false alarm rate. If conditions differ only in adopted criteria, their
ROCs will overlap. The final transfer theory, on the other hand, predicts nonoverlapping ROCs for the different conditions contrasted in Experiment 1.

To make this prediction clear, consider again the process of deciding whether or not a particular character $\alpha$ is present in the display. Ideally, as previously discussed, $\alpha$ would be reported as present whenever, prior to LCS, evidence in its favor exceeds a criterion. The ideal ROC would be traced out by moving this criterion along the evidence scale. Through competition for LCS, however, there will be a proportion $p$ of trials on which $\alpha$ is not reported, even though, prior to LCS, evidence in its favor exceeds criterion. The question of interest concerns the effect on the ROC of variations in $p$.

If $p$ is increased but criterion held constant, both hit rate and false alarm rate will decline (cf. predictions for Experiment 1). To return false alarm rate to its original value, the criterion could be reduced. But it is a general truth that the lower is criterion, the lower is the ratio of hits to false alarms. (This is reflected in the fact that ROCs are negatively accelerated.) Thus, when false alarm rate is returned to its original value, hit rate will not be. An increase in p produces a lower hit rate for a given false alarm rate.

Thus, predictions originally made for hit rates follow also for ROCs. The ROC in partial report should lie above that in whole report. In partial report, the ROC for displays with one target should lie above that for displays with three targets. These predictions were tested in Experiment 2.

\section{Exposure Conditions}

The main pattern of results in Experiment 1 held across exposure conditions. It was previously suggested that, when exposure duration is reduced to the point at which only two or three characters are ever reported, it becomes plausible to assume that performance is limited entirely by the events of stimulus input, with losses at output minimal or nonexistent. In the whole of Experiment 1, there was only a single trial on which, at the $60-$ or 90 -msec exposure durations, the subject reported more than three characters. This increases confidence that events of stimulus input determined the pattern of partial report superiority.

\section{Character Sets}

There was no evidence in Experiment 1 of a difference between character sets 1 and 2 . The separation of digits from letters prior to LCS seems not to require the presence of an obvious distinguishing feature such as a vertical line. Set 1 was used again in Experiment 2, while set 2 was dropped.

As regards set 3 , while results were basically similar to those obtained with sets 1 and 2 , in absolute size partial report superiority was reduced. At least 
this was true for one-digit displays with masked presentations. How should this effect of increased confusability between characters be viewed?

It is worth noting that partial report superiority can also be reduced by decreasing exposure duration, a manipulation which, in some ways, might be analogous to increasing confusability. Although, as regards Experiment 1, the evidence was unclearpartial report superiority was greater for unmasked than for masked cases, but among the latter was independent of exposure duration-in four other, unpublished experiments I have observed this effect of exposure duration on partial report superiority both in the comparison of unmasked and masked cases and in the effect of exposure duration in masked cases.

Accordingly, it is possible that, by increasing exposure duration for set 3 , we could produce results comparable to those of set 1 . Although there was little evidence of this in Experiment 1, a further attempt was made in Experiment 2. Pilot work suggested that exposure durations of 90 and $150 \mathrm{msec}$, respectively, for sets 1 and 3 would produce similar overall levels of performance.

\section{Category Effects in Whole Report}

In Experiment 1, performance in whole report was better for digits than for letters. In the unmasked case, this was shown by a lower false alarm rate; in the masked case, by a higher hit rate.

One possibility is that the digits were simply more discriminable. Another is that they benefited from having been the targets in partial report. To distinguish these hypotheses, half the subjects in Experiment 2 had digits as targets in partial report and letters as nontargets, while half had letters as targets and digits as nontargets.

\section{EXPERIMENT 2}

\section{Method}

\section{Subjects}

Twenty-four subjects, aged between 18 and 41 , were recruited as before. Sixteen were female.

\section{Character Sets and Target Classes}

Set 1 was used for 12 subjects, and set 3 for the remaining 12. For 6 subjects in each group, targets in partial report were digits and nontargets were letters, while for the remaining 6 subjects, targets in partial report were letters and nontargets were digits. Throughout the discussion, "targets" will refer to characters from the class defined (for the particular subject) as targets in partial report, and similarly for "nontargets."

\section{Task}

There were several changes from Experiment 1. Half the displays had one target and five nontargets, while half had three targets and three nontargets. Exposure durations were fixed at $90 \mathrm{msec}$ for character set 1 and at $150 \mathrm{msec}$ for set 3 . Displays were always immediately followed by a backward mask, lasting for $500 \mathrm{msec}$. The mask was similar in dimensions to that used before, but differed in the character presented at each of the six array positions. Instead of a superimposed 0 and + , a new character of meaningless contours was created, including horizontal, vertical, and oblique lines, with a total of 34 dots illuminated in the standard $9 \times 13$ matrix (as compared with a mean of 28 for a single digit or letter). As far as possible, this masking character resembled no particular alphanumeric character.

Each of the two tasks, partial report and whole report, was performed at three different confidence levels: cautious ("report an item only if you are absolutely sure it was there"), neutral ("not completely sure"), and risky ("report anything you have any idea could have been there").

\section{Design}

For each subject, there were six blocked experimental conditions, 2 tasks $\times 3$ confidence levels. In each of two sessions (between $1 / 2$ and $1 \frac{1}{2} \mathrm{~h}$ each), on different days, the subject performed one block of trials per condition. In the first session, each block had 52 trials, and in the second, 148. The only data analyzed were those from the last 144 trials of each secondsession block. Orders of tasks and of confidence levels were counterbalanced as before, substituting confidence levels for exposure conditions in Experiment 1. Constraints on the trial sequence, over the last 144 trials of each second-session block, were analogous to those for the last 72 trials of each such block in Experiment 1.

\section{Instructions}

Instructions were much as before, except for the careful explanation of using different confidence levels. During the practice session, attempts were made to ensure adoption of three levels noticeably different from one another.

\section{Results}

\section{Pooled Curves}

One summary of the data is provided by Figure 3. There is a separate panel for each type of display (one-target vs. three-target) for each of the two character sets. For half the subjects contributing to each panel, the targets were digits; for the other half, they were letters. Each panel has three curves, one
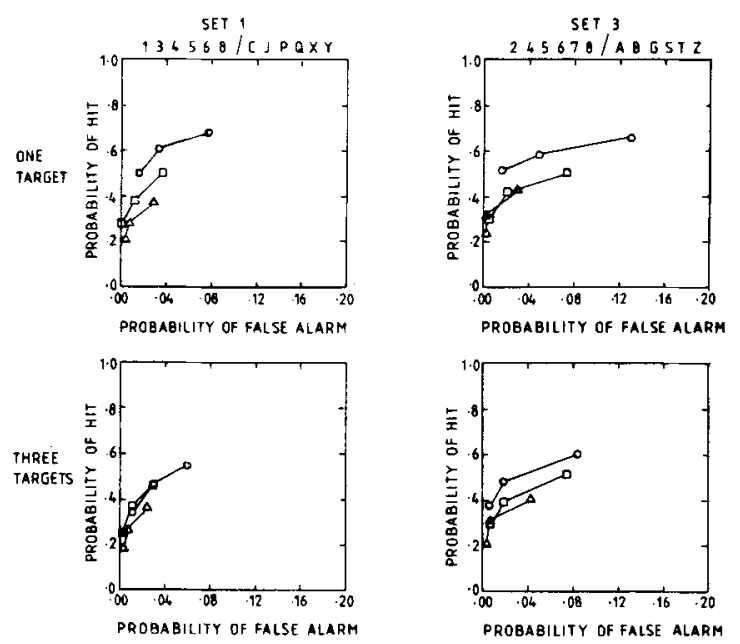

Figure 3. Experiment 2. Pooled ROC curves for each condition. $\mathrm{O}-$, targets in partial report. $\square-$, targets in whole report. $\Delta$ 一, nontargets in whole report. 
for each of three different types of report (targets in partial report, targets in whole report, nontargets in whole report).

The curves are pooled ROCs, or plots of hit rate against false alarm rate. Note that hit and falsealarm scales differ by a factor of five. The lowest, middle, and highest points of each curve show, respectively, mean hit and false alarm rates in "cautious," "neutral," and "risky" conditions.

Figure 3 provides a useful overview of the data. It shows the extent to which subjects successfully varied response criteria. It suggests very similar overall levels of performance for character sets 1 and 3. Some trends are also discernible in the data. As predicted, hit rate for a given false alarm rate was higher in partial report than in whole report. Thus partial report superiority was greater for onetarget displays than for three-target displays. Finally, it is interesting to note that, in whole report, ROCs for targets lay in three cases out of four a little above those for nontargets.

There is, however, a need for caution. Pooled ROCs produced in this way need not accurately reflect results as they hold at the level of the individual subject (McNichol, 1972). Some direct analysis of the ROCs of individual subjects is required.

\section{Individual Subjects' Curves}

Targets in partial report and whole report. For this analysis, each subject contributed four separate ROC curves-2 (partial report vs. whole report) $\times 2$ (one-target displays vs. three-target displays)-with three points per curve. A typical subject's data are shown in Figure 4. Before analysis, each curve was rendered monotonically increasing by averaging together points which: (1) shared the same hit or false alarm rate, or (2) were such that the point with a higher hit rate had a lower false alarm rate. In Figure 4, for example, the curve for partial report, three-target displays has only two points. The lower one was obtained by averaging together points for the "cautious" and "neutral" conditions, which had respective hit rates of .364 and .530 and respective false alarm rates of .0093 and .0093 .

An ideal method of analysis proved hard to find. Functions based on standard assumptions of signal detection theory (normal distributions of equal variance for "noise alone" and "signal + noise" trials) did not fit the data. Accuracy could not be measured by the standard area under the ROC curve, since, for reasons discussed in the Appendix, this measure was potentially biased in favor of partial report. Instead, a modification of the standard area measure was developed. Its disadvantage is that it results in the loss of some data, but its advantage,

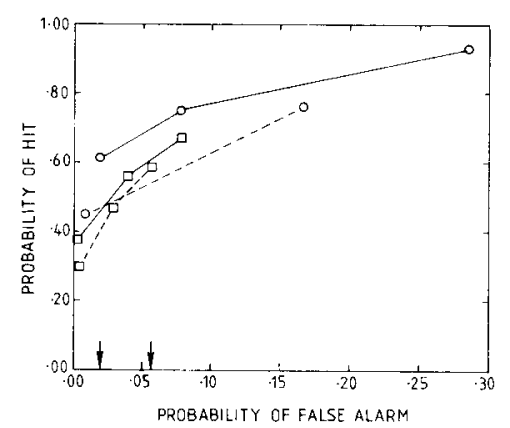

Figure 4. Experiment 2. ROC curves for a single subject. $O-$, partial report, one-target displays. $\mathrm{O} .-$, partial report, threetarget displays. $\square-$, whole report, one-target displays. $\square--$, whole report, three-target displays.

as shown in the Appendix, is that it is demonstrably not biased in favor of the experimental hypothesis.

The technique was as follows. Each of a subject's four ROC curves covered some range of false alarm rates. For example, in Figure 4 the curve for partial report, one-target displays covers the range .0194 to .2861 . Thus, the range common to all four curves could be determined; in Figure 4, it runs from .0194 to .0556 , as shown by vertical arrows on the abscissa. A subject with no common range was dropped from the analysis. Providing a common range did exist, a mean height (i.e., a mean hit rate) over the common range was obtained for each curve by finding the area under the curve over this range and dividing by range width. In Figure 4 , mean heights are $.654, .504, .541$, and .508 for, respectively: partial report, one-target displays; partial report, threetarget displays; whole report, one-target displays; and whole report, three-target displays. For each subject, the analysis thus produced a mean hit rate for each condition, with false alarm rate equated across conditions. Means across subjects are shown, separately for the four subject groups, in Table 2. The table also shows the number of subjects contributing to the analysis, and mean values of the common range.

Results were as predicted. Values of mean hit rate over the common range were higher in partial report than in whole report. In partial report, onetarget displays had a clear advantage over threetarget displays, while, in whole report, this difference was inconsistent. Thus, partial report superiority was also greater for one-target displays. These results confirm the impressions gained from Figure 3.

The data were examined by ANOVA, with character set and target class (digits vs. letters) as between-subjects factors and with task (partial report vs. whole report) and display type (one-target vs. three-target) as within-subjects factors. As predicted, there were significant effects of task $[F(1,14)$ 
Table 2

Experiment 2. Targets in Partial Report and Whole Report

\begin{tabular}{|c|c|c|c|c|c|c|}
\hline \multirow[b]{2}{*}{ Targets } & \multicolumn{2}{|c|}{ One-Target Displays } & \multicolumn{2}{|c|}{ Three-Target Displays } & \multirow{2}{*}{$\begin{array}{l}\text { Number } \\
\text { of Subjects }\end{array}$} & \multirow{2}{*}{$\begin{array}{l}\text { Common } \\
\text { Range }\end{array}$} \\
\hline & Partial Report & Whole Report & Partial Report & Whole Report & & \\
\hline & \multicolumn{6}{|c|}{ Set 1} \\
\hline Digits & .500 & .369 & .417 & .354 & 4 & $.0082-.0239$ \\
\hline \multirow[t]{2}{*}{ Letters } & .524 & .442 & .364 & .349 & 6 & $.0040-.0121$ \\
\hline & \multicolumn{6}{|c|}{ Set 3} \\
\hline Digits & .516 & .405 & .473 & .419 & 5 & $.0093-.0570$ \\
\hline Letters & .568 & .507 & .535 & .476 & 3 & $.0343-.1148$ \\
\hline
\end{tabular}

Note-The table shows mean hit rates over a common range of false alarm rates. Of the six subjects per group tested, only those with a common range were included in the analysis. Values shown under "Common Range" are means, across subjects, of start and end points of the common range.

$=23.0, \mathrm{p}<.001]$, display type $[\mathrm{F}(1,14)=42.0, \mathrm{p}<$ $.001]$, and task $\times$ display type $[F(1,14)=10.9$, $\mathrm{p}<.01]$. Two last significant interactions were character set $\times$ display type $[\mathrm{F}(1,14)=10.9, \mathrm{p}<.01]$ and target class $\times$ display type $[F(1,14)=6.7, p<$ $.05]$. Effects of display type were greater for character set 1 than for set 3 , and greater for letters than for digits, results which held in both partial report and whole report.

There is a suggestion in Table 2 that the interaction of task $x$ display type may have been weaker for character set 3 than for set 1 . However, the interaction of character set $\times$ task $\times$ display type did not approach significance $(F<1)$.

Targets and nontargets in whole report. The entire analysis just described was repeated to compare performance on targets and nontargets in whole report. Again, each subject contributed four ROC curves -2 character types $\times 2$ display types - and for these four a common range was established as before, and hence a mean hit rate for each curve across this range. Means of the values thus obtained are shown, for the four different groups of subjects, in Table 3.

For subjects whose targets were digits, performance for targets (digits) and nontargets (letters) was very similar, with nontargets, if anything, showing a slight advantage. But for subjects whose tar- gets were letters, performance for targets (letters) was very much better than for nontargets (digits).

The data were examined by ANOVA, with character set and target class as between-subjects factors and with character type (targets vs. nontargets) and display type (one-target vs. three-target) as within-subjects factors. In line with the above description, there were significant effects of character type $[F(1,13)=9.8, p<.01]$ and target class $\times$ character type $[F(1,13)=16.5, p<.01]$. Three other effects were also significant: the main effect of display type $[F(1,13)=10.6, p<.01]$ and the interactions of target class $\times$ display type $[F(1,13)=$ $7.4, p<.05]$ and character type $\times$ display type $[F(1,13)=4.8, p<.05]$. Overall, performance was a little better for one-target displays than for threetarget displays; but this effect was confined to subjects whose targets were letters, and even for them showed up only in the nontarget data.

\section{Discussion}

\section{Partial Report Superiority}

Again, the major predictions of the final transfer theory were confirmed. Performance was better in partial report than in whole report. In partial report, performance was better with one-target displays than with three-target displays, while, in whole

Table 3

Experiment 2. Targets and Nontargets in Whole Report

\begin{tabular}{|c|c|c|c|c|c|c|}
\hline \multirow[b]{2}{*}{ Targets } & \multicolumn{2}{|c|}{ One-Target Displays } & \multicolumn{2}{|c|}{ Three-Target Displays } & \multirow{2}{*}{$\begin{array}{l}\text { Number } \\
\text { of Subjects }\end{array}$} & \multirow{2}{*}{$\begin{array}{c}\text { Common } \\
\text { Range }\end{array}$} \\
\hline & Targets & Nontargets & Targets & Nontargets & & \\
\hline & \multicolumn{6}{|c|}{ Set 1} \\
\hline \multirow[t]{2}{*}{ Letters } & $\begin{array}{l}.318 \\
.339\end{array}$ & $\begin{array}{l}.324 \\
.228\end{array}$ & $\begin{array}{l}.338 \\
.315\end{array}$ & $\begin{array}{l}.345 \\
.172\end{array}$ & $\begin{array}{l}3 \\
4\end{array}$ & $\begin{array}{l}.0062-.0324 \\
.0006-.0072\end{array}$ \\
\hline & \multicolumn{6}{|c|}{ Set 3} \\
\hline $\begin{array}{l}\text { Digits } \\
\text { Letters }\end{array}$ & $\begin{array}{l}.361 \\
.334\end{array}$ & $\begin{array}{l}.405 \\
.288\end{array}$ & $\begin{array}{l}.361 \\
.347\end{array}$ & $\begin{array}{l}.371 \\
.215\end{array}$ & $\begin{array}{l}5 \\
5\end{array}$ & $\begin{array}{l}.0070-.0324 \\
.0130-.0250\end{array}$ \\
\hline
\end{tabular}

Note-The table shows mean hit rates over a common range of false alarm rates. Of the six subjects per group tested, only those with a common range were included in the analysis. Values shown under "Common Range" are means, across subjects, of start and end points of the common range. 
report, the suggestion of a similar effect of display type was much weaker. Thus, partial report superiority was also greater with one-target displays. Confirmation of all these results in the ROCs of individual subjects shows they cannot be ascribed to simple criterion shifts.

\section{Output Effects}

Again, a further analysis was conducted to check the importance of output effects. In the whole of Experiment 2, there were 4 trials (contributed by a single subject) on which more than three items were reported in the "cautious" condition, 55 (contributed by five subjects), or $1.5 \%$ of all trials, in the "neutral" condition. Yet, when the analysis of individual subjects' ROCs was repeated, ${ }^{2}$ dropping the "risky" condition from consideration, mean advantages of partial report over whole report were .127 and .065 , respectively, for one-target and threetarget displays, values comparing favorably with those shown in Table 2. Again, this analysis increases confidence that the pattern of results was determined by events of stimulus input, rather than by losses during output.

\section{Character Sets}

In Experiment 2, the main difference between character sets was a suggestion that, for set 3 , the interaction between task (partial report vs. whole report) and display type (one-target vs. three-target) might be reduced. Although this reduction was not significant, it might repay further investigation.

In general, however, it seems fair to conclude that the basic pattern of results was similar for all three character sets used in the present work.

\section{Whole Report}

In Experiment 1, performance in whole report was better for digits than for letters. Two hypotheses were (1) that digits were more discriminable, and (2) that digits gained through having been targets in partial report. The results of Experiment 2 support the second hypothesis. For subjects whose targets were digits, performance in whole report was similar for targets and nontargets. For subjects whose targets were letters, performance in whole report was better for targets. Although these data are rather untidy, I have confirmed in several other experiments that the characters serving as targets in partial report will usually show an advantage in whole report.

In terms of the final transfer theory, an obvious explanation is that even in whole report there may be a bias, when choosing which characters to pass into LCS, towards characters that have been targets in partial report. Earlier, we defined $\mathbf{p}$ as the probability, through competition for LCS, that a character $a$ will not be reported even though prior to LCS evidence in its favor exceeds criterion. The present hypothesis is that, in whole report, $p$ may be lower (and hence performance better) for targets than for nontargets.

The same proposal could account for a second unexpected result in Experiments 1 and 2. Even in whole report there was a tendency for performance to be better with one-target displays than with threetarget displays. Sometimes this result held for targets, sometimes for nontargets. If a character $\alpha$ shares the display with $\mathrm{x}$ targets and $\mathrm{y}$ nontargets, and if targets compete more strongly for LCS, then, for $\alpha, p$ will decrease (and hence performance will improve) as $\mathrm{x}$ decreases and $\mathrm{y}$ increases.

\section{GENERAL DISCUSSION}

The present research has produced two main findings. Reporting digits (letters) from a display is more succesful if letters (digits) can be ignored than if they cannot. If letters (digits) can be ignored, reporting digits (letters) is more successful the fewer the digits (letters) in the display. Like the results of Duncan (1980), these findings suggest that a character's access to LCS can be determined by its alphanumeric class. They thus support the proposition, embedded in the final transfer theory, that even a detailed analysis of form and aspects of meaning can precede LCS.

Here three alternative explanations for some or all of the results will be considered.

\section{The Difficulty of Interpreting Interactions}

A crucial finding was that the difference in performance between partial report and whole report was greater for one-target displays than for threetarget displays. Interactions of this sort can be hard to interpret. For example, even in whole report, performance was sometimes better for one-target displays than for three-target displays. If performance for these two had been equated in whole report, the interaction might have disappeared.

This doubt may be removed by considering the effects of exposure duration. An increase in exposure duration (and hence in the level of performance) for three-target displays does not produce partialreport superiority equal to that obtained, even at a lower exposure duration and thus comparable level of whole-report performance, for one-target displays. This is clear in Figure 1 and is confirmed also in my other, unpublished partial-report studies.

\section{Confusability Within and Between Classes}

Consider again the problem of an ideal observer's deciding, for each display, whether or not a particular character $\alpha$ is present. In the earlier discussion, it was assumed that the average confusability between $\alpha$ and digits was equal to the average confusability between $\alpha$ and letters. In this case, 
performance will not depend on how many of the displayed characters are digits and how many are letters.

Suppose, however, that when $\alpha$ is a digit, it is more confusable with other digits than with letters, and that when $\alpha$ is a letter, it is more confusable with other letters than with digits. For the ideal observer, it will be harder to discriminate between displays with and without $\alpha$, the more similar to $\alpha$ are other displayed characters. Given the new confusability assumption, this will mean that performance declines as the number of displayed characters from the same class as $\alpha$ (digits or letters) increases.

An account of this sort might well explain why, for target characters, performance is better with one-target displays than with three-target displays. There are, however, two grounds on which the account fails. First, it offers no explanation for partial report superiority, or for the increased effect of display type in the partial report task. Second, it predicts that performance for nontargets should be better with three-target displays than with onetarget displays, the reverse of the obtained result.

\section{Partial Shape Analysis}

The last alternative explanation accepts that, to some extent at least, digits and letters can be separated prior to LCS. It denies, however, that, at this level, characters are already fully identified. Instead, the suggestion is that digits and letters can be separated on the basis of some partial analysis of key stimulus features (Treisman \& Gelade, 1980). Though it is hard to imagine what the features might be, the issue remains open as long as they are unspecified.

An extension of this view is particularly interesting. The present data do not show that digits and letters can be told perfectly apart prior to LCS. Accuracy on a given character will be higher, the less strongly other presented characters compete for LCS. With this in mind, the results do not show that, in partial report, nontargets are kept out of LCS entirely. They show only that nontargets compete for LCS less strongly in partial report than in whole report, and that in partial report nontargets compete less strongly than targets. This alone would give higher accuracy for targets in partial report than in whole report and, in partial report, higher accuracy for displays with fewer targets. Yet a selection schedule reducing only the probability of LCS access for the class of nontargets as a whole (and perhaps only for some nontargets), and increasing it only for the class of targets as a whole (and perhaps only for some targets), could evidently be based on only a partial shape analysis.

The strongest evidence against a position of this kind comes from experiments suggesting that even the meaning of an auditorily presented word can determine LCS access (Ostry et al., 1976), surely requiring complete preattentive word identification. Other arguments, however, directly concern the separation of digits and letters.

First, if preattentive separation of digits from letters is based on a partial analysis of key features, it is surprising that it does not improve when a single feature is sufficient for perfect separation. Yet, in Experiment 1, performance was very similar with character sets 1 and 2 . Here we should have to suppose that the system is inflexible, failing to profit from the special opportunities offered by particular digit and letter subsets.

Second, if preattentive separation of digits from letters is based on partial analysis of key features, it is surprising that unfamiliar character sets cannot be similarly separated. For example in one of the experiments of Duncan (1980), subjects searched for targets from the set $\mathbf{8 G}$ appearing among nontargets from the set 3569 COSQ. If features of sufficient complexity can be found to separate 134568 from CJPQXY (the present set 1), surely they could be found to separate $8 \mathrm{G}$ from $3569 \mathrm{COSQ}$. Yet the results of Duncan's experiment suggested no ability to select only targets for LCS access (in line with the final transfer theory's postulate that only welllearned categorizations can precede LCS). Here we should have to suppose that features distinguishing two sets are found only after long practice on the distinction.

Third, in the present work it was not true that partial report superiority held only for some members of each target set. In Experiment 1, hit rates were higher in partial report than in whole report for each individual target in all three target sets. In Experiment 2, by criteria like those used before, ROCs lay higher in partial report than in whole report for each individual target in all four target sets, with only one exception. If selection schedules in partial report increased the probability of LCS access only for some targets, then different targets must have been favored either across trials or across subjects.

\section{REFERENCE NOTE}

1. Duncan, J. Partial reports based on color and on alphanumeric class: Evidence for a late selection theory of attention. Unpublished manuscript, 1979.

\section{REFERENCES}

Allport, D. A. Parallel encoding within and between elementary stimulus dimensions. Perception \& Psychophysics, 1971, 10, 104-108.

Allport, D. A. On knowing the meaning of words we are unable to report: The effects of visual masking. In S. Dornic (Ed.), Attention and performance VI. Hillsdale, N.J: Erlbaum, 1977. 
Broadbent, D. E. Decision and stress. London: Academic Press, 1971.

Dick, A. O. Relations between the sensory register and shortterm storage in tachistoscopic recognition. Journal of Experimental Psychology, 1969, 89, 279-284.

Dick, A. O. Visual processing and the use of redundant information in tachistoscopic recognition. Canadian Journal of Psychology, 1970, 24, 133-141.

Dick, A. O. On the problem of selection in short-term visual (iconic) memory. Canadian Journal of Psychology, 1971, 25, 250-263.

Duncan, J. The locus of interference in the perception of simultaneous stimuli. Psychological Review, 1980, 87, 272-300.

Duncan, J. Directing attention in the visual field. Perception \& Psychophysics, 1981, 30, 90-93.

McNicol, D. A primer of signal detection theory. London: Allen \& Unwin, 1972.

MerikLe, P. M. Selection from visual persistence by perceptual groups and category membership. Journal of Experimental Psychology: General, 1980, 109, 279-295.

Moray, N., Fitter, M., Ostry, D., Favaeau, D., \& Nagy, V. Attention to pure tones. Quarterly Journal of Experimental Psychology, 1976, 28, 271-283.

Ostry, D., Moray, N., \& Marks, G. Attention, practice, and semantic targets. Journal of Experimental Psychology: Human Perception and Performance, 1976, 2, 326-336.

Posner, M. I. Chronometric explorations of mind. Hillsdale, N.J: Erlbaum, 1978.

Poulton, E. C. The measurement of legibility. Printing Technology, 1968, 12, 2-6.

Schneider, W., \& Shiffrin, R. M. Controlled and automatic human information processing: $\mathrm{I}$. Detection, search, and attention. Psychological Review, 1977, 84, 1-66.

Shaw, M. L. Attending to multiple sources of information: I. The integration of information in decision making. Cognitive Psychology, 1982, 14, 353-409.

Sorkin, R. D., Pohlmann, L. D., \& Gilliom, J. Simultaneous two-channel signal detection: III. 630 and $1400 \mathrm{~Hz}$ signals. Journal of the Acoustical Society of America, 1973, 53, 10451051.

SPERLiNG, G. The information available in brief visual presentations. Psychological Monographs, 1960, 74(11, Whole No. 498).

SPERLING, G. Successive approximations to a model for shortterm memory. In A. F. Sanders (Ed.), Attention and performance I. Amsterdam: North-Holland, 1967.

TAYLOR, D. A. Identification and categorization of letters and digits. Journal of Experimental Psychology: Human Perception and Performance, 1978, 4, 423-439.

Treisman, A., \& Gelade, G. A feature-integration theory of attention. Cognitive Psychology, 1980, 12, 97-136.

von Wright, J. M. Selection in visual immediate memory. Quarterly Journal of Experimental Psychology, 1968, 20, 62-68.

voN WRIGHT, J. M. On selection in visual immediate memory. In A. F. Sanders (Ed.), Attention and performance III. Amsterdam: North-Holland, 1970.

voN Wright, J. M. On the problem of selection in iconic memory. Scandinavian Journal of Psychology, 1972, 13, 159-171.

\section{NOTES}

1. I am indebted to Donald Broadbent for raising this point.

2 . The original analysis was slightly revised. Instead of finding a common range for all four of a subject's ROCs, separate common ranges were used for one-target displays and threetarget displays, each given by the overlap of ranges for partial report and whole report. In this way, it was possible, even dropping the "risky" condition, to keep 16 of the original 24 subjects in the analysis.

\section{APPENDIX}

In Figure 5 is drawn a "true" ROC curve (dotted) and the empirical (solid) curve that would be produced by establishing experimentally three points $(A, B, C)$ on the true curve. Since, in the empirical curve, the three points are joined by straight lines (and by straight lines to the bottom left and top right corners of the graph), the area under this empirical curve is less than the area under the true curve.

The extent of this downward bias depends on the spread of the established points along the true curve. Imagine the three points all clustered together around the position of the drawn point $A$. Even though all three points might lie on the true curve, the area under the new empirical curve would obviously be much smaller than the area under the empirical curve drawn.

For this reason, the standard area measure (McNicol, 1972) was inappropriate for the data of Experiment 2. As the spread of points was typically greater for partial report than for whole report, downward bias from the area under a true curve was probably smaller. An advantage of partial report over whole report could have been found artefactually. To correct for this, the standard area measure was adapted, as described in the text, to consider only that range of false alarm rates common to the ROCs under comparison.

Even with the new measure, some bias is possible. For example, one suspects that in Figure 4 the ROC for partial report, three-target displays would lie considerably higher within the common range, were more points established either within or near to this range. Because the two points of this curve lie far apart, the straight line joining them probably lies quite far below the true, continuous curve. Although there is no reason for such influences consistently to favor one condition over another, some check on this possibility is required.

To this end, a further analysis was designed, in which all possible bias works against the experimental hypothesis. Empirical ROCs are made up of two sorts of point: points established experimentally (A, B, C in Figure 5) and points lying on the interpolated straight lines. The former are presumably not biased either consistently above or consistently below the true ROC, while the latter are biased below. The new analysis, starting with a hypothesis that the ROC in condition $A$ lies above that in condition B, takes into account both types of point for.A, but only experimentally established points for $B$.

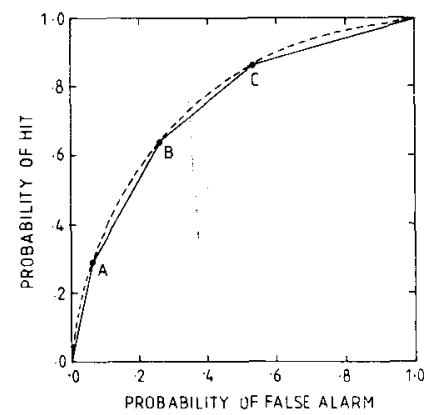

Figure 5. True (dotted) and empirical (solid) ROC curves. 
The technique may be illustrated by considering, with reference to Figure 4, the hypothesis that for one-target displays the ROC for partial report lies above that for whole report. The experimentally established points of the partial report curve are joined by straight lines, as shown in the figure. Each experimentally established point of the whole-report curve is then considered in turn. A whole-report point lying outside the range of false alarm rates covered by the partial report curve is dropped from the analysis (cf. the "cautious" point in Figure 4). For each point within the range, the vertical distance below the partial report curve is measured, and a mean then taken across points. As in the main analysis, one thus obtains a mean difference in height (hit rate) between partial report and whole report curves, with false alarm rate equated in the two cases, but with only experimentally established points considered for whole report.

This analysis was separately conducted for each subject, and the results compared with those of the main analysis. In the new analysis, the measured advantage (in hit rate) of partial report over whole report was reduced from its previous value by only .004 and .003 for one-target displays and three-target displays, respectively. In partial report, the mean advantage of one-target displays over three-target displays was reduced by .010 . In whole report, the mean advantage of targets over nontargets was reduced by .018 . Thus, even the new, conservative analysis left results essentially unchanged.

(Manuscript received April 28, 1982;

revision accepted for publication February 4, 1983.) 